\title{
Finding your practice home base
}

\author{
David H Henry, MD
}

A s summer winds down and we begin to gear up to return to school or work, I was thinking about new and returning hem-onc residents, fellows, and young attendings and a question I routinely get from them: what should I do next in my career? I always answer by holding up 3 fingers and telling them that they can practice 1 , at a university hospital; 2 , at a university teaching affiliate; or 3 , at a community hospital or practice with a little or no university affiliation. These days, trainees in hematology-oncology are often advised to be highly specialtyspecific when they plan their long-term careers and to focus on a particular cancer or hematologic disorder. That is fine if you want to remain in an academic or university-based practice, but not if community practice is your preference. So, what are the differences among these 3 options?

Option 1, to remain in a university setting where you can be highly focused and specialized in a single narrowly defined area, could be satisfying, but keep in mind that the institution expects results! You will be carefully monitored for research output and teaching and administration commitments, and your interaction with patients could add up to less than $50 \%$ of your time. Publication and grant renewal will also play a role and therefore take up your time.

If you are considering option 2 - to work at a university teaching affiliate hospital - you need to bear in mind that you likely will see a patient population with a much broader range of diagnoses than would be the case with the first option. Patient care for option 2 will take up more than $50 \%$ of your time, so it might be a little more challenging to stay current, but perhaps more refreshing if you enjoy contact with patients. Teaching, research, and administration will surely be available, and publication and grant renewal will play as big or small a role as you want.

Option 3 would be to join a community hospital or practice where the primary focus is on patient care and the diagnoses will span the hematology and oncology spectrum. This type of practice can be very demanding of one's time, but as rewarding as the other options, especially if you value contact with patients. With this option, one is more likely to practice as a generalist, perhaps with an emphasis in one of the hem-onc specialties, but able to treat a cluster of different types of cancer as well.

I always advise trainees to be sure they ask physicians practicing in each of these options to give examples of what their best and worst days are like so that they can get some idea of what the daily humdrum and challenges would encompass. What did I choose? I have always gone with option 2 and have been very happy in that setting.

In this issue...

More biosimilars head our way. Turning to the current issue of the journal, on page e181, Dr Jane de Lartigue discusses 2 new biosimilars recently approved by the United States Food and Drug Administration (FDA) - epoetin alfa-epbx (Retacrit; Hospira, a Pfizer company) for chemotherapy-induced anemia (CIA), and pegfilgrastim-jmdb (Fulphila; Mylan and Biocon) for prevention of febrile neutropenia. As Dr de Lartigue notes, biosimilars are copies of FDA-approved biologic drugs that cannot be identical to the reference drug but demonstrate a high similarity to it. In this case, the reference drug for epoetin alfa-epbx is epoetin alfa (Epogen/Procrit, Amgen) and for pegfilgrastim-jmdb, it is pegfilgrastim (Neulasta, Amgen). As the reference drugs' patents expire, biosimilars are being developed to increase competition in the marketplace in an effort to reduce costs and improve patient access to these therapies. Indeed, the FDA is working to streamline the biosimilar approval process to facilitate that access.

Reading this article got me thinking about something I often have to consider in the course of my work: transfusion versus erythropoiesis-stimulating agents (ESAs)? Recombinant erythropoietin drugs such as the biosimilar, epoetin alfa-epbx, and its reference drug are grouped together as ESAs, and have been used to treat CIA since the late 1980s. However, there were a few trials that used higherdose ESA or set high hemoglobin targets, and their findings suggested that ESAs may shorten survival in patients with cancer or increase tumor growth, or both. The use of ESAs took a nosedive after the 2007 decision by the FDA's Oncologic Drugs Advisory Committee to rein in their use for a hard start of ESA treatment at less than $10 \mathrm{~g} / \mathrm{dL}$ hemoglobin, and not higher. Subsequent trials addressed the 
concerns about survival and tumor growth. A meta-analysis of 60 randomized, placebo-controlled trials of ESAs in CIA found that there was no difference in overall survival between the study and control groups. ${ }^{1}$ Likewise, findings from an FDA-mandated trial with epoetin alfa (Procrit) in patients with metastatic breast cancer have reported that there was no significant difference in overall survival between the study and control groups. ${ }^{2}$ The results of a second FDA-mandated trial with darbepoetin alfa (Aranesp, Amgen) in patients with metastatic lung cancer are expected to be released soon. The FDA lifted the ESA Risk Evaluation and Mitigation Strategy based on those findings. However, many practitioners, both young and old, continue to shy away from using ESAs because of the FDA black box warning that remains in place despite the latest data. ${ }^{3}$

The use of transfusion ticked up reciprocally with the decline in ESA use, but perhaps we should re-evaluate the use of these agents in our practice, especially now that the less costly, equally safe and effective biosimilars are becoming available and we have the new survival data. Transfusions are time consuming and have side effects, including allergic reaction and infection risk, whereas ESAs are easily administered by injection, which patients might find preferable.

Malignancies in patients with HIV-AIDS. On page e188, Koppaka and colleagues report on a study in India of the patterns of malignancies in patients with HIV-AIDS. I began my career just as the first reports of what became known as HIV-AIDS emerged, and we were all mystified by what was killing these patients and the curious hematologic and oncologic problems they developed. Back then, the patients were profoundly immunosuppressed, and the immunosuppression cancers of non-Hodgkin lymphoma, usually higher grade, and Kaposi sarcoma were most prevalent and today are collectively labeled AIDS-defining malignancies (ADMs).

Fast forward to present day, and we have extremely effective antiretroviral therapies that have resulted in a significant reduction in mortality among HIV-infected individuals who are now living long enough to get what we call non-AIDS-defining malignancies (NADMs) such as anal or cervical cancers, hepatoma (hepatocellular carcinoma), Hodgkin lymphoma, and lung cancer. Of note is that these NADMs are all highly viral associated, with anal and cervical cancers linked to infection with the human papilloma virus; hepatoma linked to the hepatitis $\mathrm{B} / \mathrm{C}$ viruses; Hodgkin lymphoma to the Epstein-Barr virus; and lung cancer, possibly also HPV. Fortunately, these days we can use standard-dose chemoradiation therapy for all HIVrelated cancers because the patients' immune systems are much better reconstituted and the modern-day antiretroviral therapies have much less drug-drug interaction thanks to the advent of the integrase inhibitors. The researchers give an excellent breakdown of the occurrence of these malignancies, as well as an analysis of the correlation between CD4 counts and the different malignancies.

Immunotherapy-related side effects in the ED. What happens when our patients who are on immunotherapy end up in the emergency department (ED) with therapyrelated symptoms? And what can the treating oncologist do to help the ED physician achieve the best possible outcome for the patient? I spoke to Dr Maura Sammon, an ED physician, about some of the more common of these side effects - lung, gastrointestinal, rash, and endocrine-related problems - and she describes in detail how physicians in the ED would triage and treat the patient. Dr Sammon also emphasizes the importance of communication: first, between the treating oncologist and patient, about the differences between chemotherapy and immunotherapy; and second, between the ED physician and the treating oncologist as soon as possible after the patient has presented to ensure a good outcome. The interview is part of The JCSO Interview series. It is jam-packed with useful, how-to information, and you can read a transcript of it on page e216 of this issue, or you can listen to it online. ${ }^{4}$

We round off the issue with a selection of Case Reports (pp. e200-e209), an original report on the characteristics of urgent palliative cancer care consultations encountered by radiation oncologists (p. e193), and a New Therapies feature, also by Dr de Lartigue, focusing on the rarity and complexities of sarcomas (p. e210).

Those are my dog-day-of-summer thoughts as we head toward another Labor Day and a new academic year. Since we are all online now, we encourage you to listen to my bimonthly podcast of each issue on our website at www.jcsoonline.com, and of course, follow us on Twitter (@jcs_onc) and Instagram (@jcsoncology) and like us on Facebook.
References
1. Glaspy J, Crawford J, Vansteenkiste J, et al. Erythropoiesis-
stimulating agents in oncology: a study-level meta-analysis of sur-
vival and other safety outcomes. Br J Cancer. 2010;102(2):301-315.
2. Leyland-Jones B, Bondarenko I, Nemsadze G, et al. A randomized,
open-label, multicenter, phase III study of epoetin alfa versus best
standard of care in anemic patients with metastatic breast cancer
receiving standard chemotherapy. J Clin Oncol. 2016;34:1197-1207.
3. US Food and Drug Administration release. Information on erythro- poiesis-stimulating agents (ESA) epoetin alfa (marketed as Procrit, Epogen), darbepoetin alfa (marketed as Aranesp). https://www.fda. gov/Drugs/DrugSafety/ucm109375.htm. Last updated April 13, 2017. Accessed August 20, 2018.

4. Henry D, Sammon M. Treating immunotherapy-related AEs in the emergency department [Audio]. https:/www.mdedge.com/jcso/article/171966/patient-survivor-care/treating-immunotherapy-relatedaes-emergency-department. Published August 6, 2018. 